Pacific Journal of Mathematics

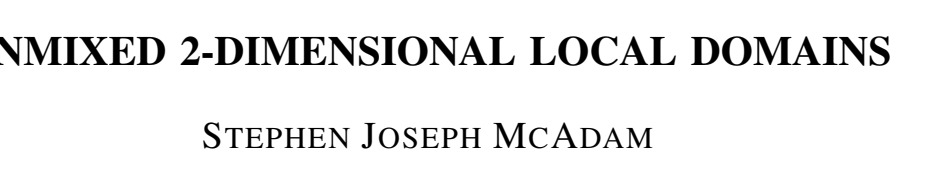




\title{
UNMIXED 2-DIMENSIONAL LOCAL DOMAINS
}

\author{
Stephen McAdam
}

\begin{abstract}
Let $b, c$ be a system of parameters in a 2-dimensional local (Noetherian) domain $(R, M)$. For $n \geqq 0$, the chain $\left(b^{n}: 1\right) \subset\left(b^{n}: c\right) \subset\left(b^{n}: c^{2}\right) \subset \cdots$ becomes stable. Thus define a function $S(b, c,-)$ by letting $S(b, c, n)$ be the least integer $k \geqq 0$ such that $\left(b^{n}: c^{k}\right)=\left(b^{n}: c^{k+1}\right)$. Ratliff has shown that $R$ is unmixed if and only if $S(b, c,-)$ is bounded. This paper shows that if $R$ is unmixed then for any $0 \neq d \in M$ there is an integer $* d \geqq 0$ such that for any system of parameters $b, c$ and any $i \geqq 0, S\left(b, c,{ }^{*} b+i\right)={ }^{*} c$.
\end{abstract}

Introduction. We consider a 2-dimensional local domain $(R, M)$ with a system of parameters $b, c$. (That is, $b$ and $c$ are nonzero nonunits, and no height 1 prime contains both of them.) For a fixed $n \geqq 0$, obviously $\left(b^{n}: 1\right) \subset\left(b^{n}: c\right) \subset\left(b^{n}: c^{2}\right) \subset \cdots$. As this chain eventually becomes stable, we define a function $S(b, c,-)$ by letting $S(b, c, n)$ be the least integer $k \geqq 0$ such that $\left(b^{n}: c^{k}\right)=\left(b^{n}: c^{k+1}\right)=\cdots$. A recent result of Ratliff shows that $R$ is unmixed if and only if $S(b, c,-)$ is bounded. In this paper we show that if $R$ is unmixed, then for any $0 \neq d \in M$ there is an integer ${ }^{*} d \geqq 0$ such that for any system of parameters $b, c$, and for any $i \geqq 0, S\left(b, c,{ }^{*} b+i\right)={ }^{*} c$.

Notation. Throughout this paper, $(\mathrm{R}, \mathrm{M})$ will be a 2 -dimensional local domain and $b, c$ will be a system of parameters for $R$. For $d \in R$, $d^{0}=1$.

We consider the following two arrays of ideals, the displayed inclusions being trivial.

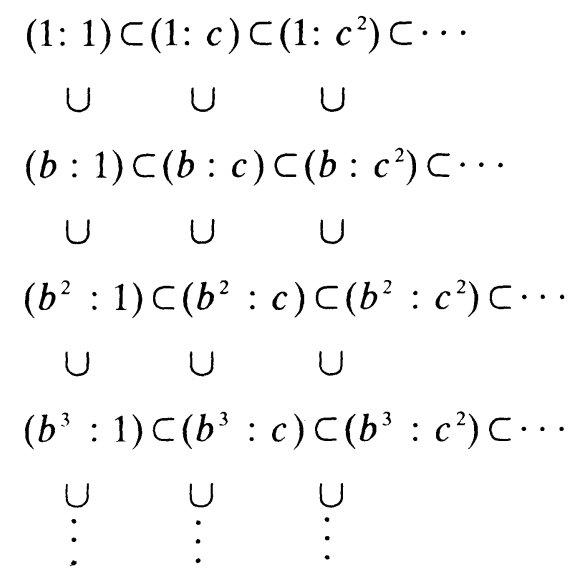


and

$$
\begin{aligned}
& (1: 1) \subset(1: b) \subset\left(1: b^{2}\right) \subset \cdots \\
& (c: 1) \subset(c: b) \subset\left(c: b^{2}\right) \subset \cdots \\
& \cup \cup \cup \\
& \left(c^{2}: 1\right) \subset\left(c^{2}: b\right) \subset\left(c^{2}: b^{2}\right) \subset \cdots \\
& \cup \quad \cup \quad \cup \\
& \left(c^{3}: 1\right) \subset\left(c^{3}: b\right) \subset\left(c^{3}: b^{2}\right) \subset \cdots \\
& \begin{array}{lll}
\cup & \cup & \cup \\
\vdots & \vdots & \vdots
\end{array}
\end{aligned}
$$

By A.C.C. it is obvious that the rows in these arrays eventually become stable. Our first lemma relates the rows of one array to the columns of the other, and shows that the columns eventually become well behaved.

LEMMA 1. The following are equivalent for $n \geqq 0$ and $m \geqq 0$

(i) $\left(b^{n}: c^{m}\right)=\left(b^{n}: c^{m+1}\right)=\cdots$

(ii) $\left(c^{m+1}: b^{n}\right) \subset(c)$

(iii) $\left(c^{m+1}: b^{n}\right)=c^{\prime}\left(c^{m}: b^{n}\right)$ for $j=1,2, \cdots$.

Proof. (i) $\Rightarrow$ (iii): Suppose that $a \in\left(c^{m+1}: b^{n}\right)$. Write $a b^{n}=$ $c^{m+1} d$. Then $d \in\left(b^{n}: c^{m+1}\right)=\left(b^{n}: c^{m}\right)$ (by i). Thus we may write $d c^{m}=b^{n} e$ where $e \in\left(c^{m}: b^{n}\right)$. Now $a b^{n}=c^{m+1} d=c^{\prime} b^{n} e$ so that $a=$ $c^{\prime} e$ giving $a \in c^{\prime}\left(c^{m}: b^{n}\right)$.

(iii) $\Rightarrow$ (ii): Immediate.

(ii) $\Rightarrow$ (i): Suppose that $f \in\left(b^{n}: c^{m+1}\right)$ and write $c^{m+1} f=b^{n} g$. Then $g \in\left(c^{m+1}: b^{n}\right) \subset(c)$ (by ii). We write $g=c h$. Then $c^{m+1} f=b^{n} g=b^{n} c h$ so that $c^{m} f=b^{n} h$. Thus $f \in\left(b^{n}: c^{m}\right)$. This shows that $\left(b^{n}: c^{m}\right)=$ $\left(b^{n}: c^{m+1}\right)$ form which it follows easily that $\left(b^{n}: c^{m}\right)=\left(b^{n}: c^{m+1}\right)$ for all $i \geqq 0$.

For each of the two arrays above we define two functions. Considering the first array, for $n \geqq 0$, let $S(b, c, n)$ be the least integer $k \geqq 0$ such that $\left(b^{n}: c^{k}\right)=\left(b^{n}: c^{k+1}\right)=\cdots$. (Obviously $S(b, c, 0)=0$.) For $m \geqq 0$ let $C(b, c, m)$ be the least integer $l \geqq 1$ such that $\left(b^{l}: c^{m}\right) \subset(b)$. (Obviously $C(b, c, 0)=1$.) Of course $S(c, b,-)$ and $C(c, b,-)$ are defined analogously.

LEMma 2. $R$ is Macaulay if and only if $S(b, c,-)$ is identically 0. 
Proof. $\quad\left(b: c^{0}\right)=(b: 1)=(b)$, while $(b: c)=(b)$ exactly when $b, c$ is an $R$-sequence, i.e., exactly when $R$ is Macaulay. The result is now obvious.

LeMmA 3. $C(b, c,-)=S(c, b,-)+1$.

Proof. For $m \geqq 0$ let $C(b, c, m)=l_{1}$ and $S(c, b, m)=l_{2}$. Since $\left(b^{0}: c^{m}\right)=\left(1: c^{m}\right)=R \not \subset(b)$, clearly $l_{1} \geqq 1$. By the definition of these functions we have $\left(b^{l_{1}}: c^{m}\right) \subset(b)$ and $\left(c^{m}: b^{h}\right)=\left(c^{m}: b^{h^{+1}}\right)=\cdots$. By Lemma $1,\left(c^{m}: b^{l_{1}-1}\right)=\left(c^{m}: b^{l_{1}}\right)=\cdots$ and $\left(b^{l^{+1}}: c^{m}\right) \subset(b)$. By the minimal conditions in the definitions of our functions, it follows that $l_{2} \leqq l_{1}-1$ and $l_{1} \leqq l_{2}+1$. Thus $l_{1}=l_{2}+1$.

LEMMA 4. $C(b, c,-)$ and $S(b, c,-)$ are both monotonically increasing.

Proof. By Lemma 3 and symmetry it is enough to show that $C(b, c,-)$ is monotonically increasing. For this, say that $C(b, c, m+1)$ $=l$. Then $\left(b^{l}: c^{m+1}\right) \subset(b)$. However $\left(b^{l}: c^{m}\right) \subset\left(b^{l}: c^{m+1}\right) \subset(b)$ showing that $C(b, c, m) \leqq l=C(b, c, m+1)$.

We wish to translate a result of Ratliff into our language. Ratliff uses the ideal $(b R)^{(k)}=\{r \in R \mid$ there is an $s \in R$ with $s$ in no minimal prime divisor of $b$, such that $\left.s r \in\left(b^{k}\right)\right\}$. We have a preliminary lemma.

LEMma 5. If $S(b, c, k)=l$ then $(b R)^{(k)}=\left(b^{k}: c^{l}\right)$.

Proof. If $R$ is Macaulay, both of these ideals are just $\left(b^{k}\right)$. If $R$ is not Macaulay, choose $n \geqq l$ such that $c^{n}$ is in an $M$-primary component of $\left(b^{k}\right)$. It is easy to verify that $(b R)^{(k)}=\left(b^{k}: c^{n}\right)$. However $n \geqq l=$ $S(b, c, k)$ says that $\left(b^{k}: c^{n}\right)=\left(b^{k}: c^{l}\right)$. Thus $(b R)^{(k)}=\left(b^{k}: c^{l}\right)$.

We now treat Ratliff's result. Recall that $R$ is unmixed means that in the completion of $R$, each prime divisor of zero has depth equal to $\operatorname{dim} R$ ( $=2$ in our case).

Proposition 6. The following are equivalent.

(i) $R$ is unmixed

(ii) $S(b, c,-)$ is bounded

(iii) $C(b, c,-)$ is bounded.

Proof. The equivalence of (i) and (ii) is immediate from $[2,3.6 .1 \Leftrightarrow$ 3.6.2] and Lemma 5. The equivalence of (ii) and (iii) is by Lemma 3. 
REMARK. Following Theorem 9 we will outline a second proof of Proposition 6.

COROLlary 7. R is unmixed if and only if $S(b, c,-)$ and $C(b, c,-)$ are eventually constant.

Proof. Immediate from Proposition 6 and Lemma 4.

We now begin showing that if $R$ is unmixed, then the constant which $S(b, c,-)$ eventually becomes depends only on $c$. To do so we make use of $R^{(1)}=\cap R_{P}$ over all height 1 primes $P$.

LEMma 8. $R^{(1)}=\left\{a / b^{n} \mid a \in\left(b^{n}: c^{m}\right)\right.$ for some $n$ and $\left.m\right\}$.

Proof. Suppose that $x \in R^{(1)}$ and let $I=\{r \in R \mid r x \in R\}$. As $x \in R^{(1)}=\cap R_{P}$ over all height 1 primes $P, I$ is not contained in any height 1 prime. Since $\operatorname{dim} R=2$, we have $\operatorname{rad} I=M$ and so for some $n$ and $m, b^{n} \in I$ and $c^{m} \in I$. We write $b^{n} x=a \in R$ and $c^{m} x=$ $d \in R$. We see that $a c^{m}=d b^{n}$. Thus $x=a / b^{n}$ with $a \in\left(b^{n}: c^{m}\right)$.

Conversely if $a \in\left(b^{n}: c^{m}\right)$ write $a c^{m}=b^{n} d$. If $x=a / b^{n}=d / c^{m}$ then $b^{n}$ and $c^{m}$ are in $I=\{r \in R \mid r x \in R\}$. As $b, c$ is a system of parameters, no height 1 prime can contain $I$. Thus $x \in R^{(1)}$.

THEOREM 9. $C(b, c,-)$ is bounded if and only if $R^{(1)}$ is a finitely generated $R$-module. In fact if $C(b, c,-)$ is bounded and eventually equals $k+1$, then $k$ is the least integer such that $b^{k} R^{(1)} \subset R$.

Proof. Suppose that $C(b, c,-)$ is bounded. By Lemma 4 it is eventually constant, say at $k+1$. Of course by Lemma $3, k \geqq 0$. We first show that $b^{k-1} R^{(1)} \not \subset R$. Since $C(b, c,-)$ is eventually $k+1$, for some $l$ we have $C(b, c, l)=k+1$. That is, $\left(b^{k+1}: c^{l}\right) \subset(b)$ but $\left(b^{k}: c^{l}\right) \not \subset(b)$. Choose $a \in\left(b^{k}: c^{l}\right)-(b)$. By Lemma 8, $a / b^{k} \in$ $R^{(1)}$. However $a \notin(b)$ showing $b^{k-1}\left(a / b^{k}\right) \notin R$. Thus $b^{k-1} R^{(1)} \not \subset R$.

We now show that $b^{k} R^{(1)} \subset R$. Choose $x \in R^{(1)}$ and write $x=a / b^{n}$ with $a \in\left(b^{n}: c^{m}\right)$ for some $n$ and $m$. If $n \leqq k$ then clearly $b^{k} x \in R$. Thus suppose that $n=k+j$ for some $j \geqq 1$. If $C(b, c, m)=$ $k_{1}$ then $\left(b^{k_{1}}: c^{m}\right) \subset(b)$ and since $C(b, c,-)$ monotonically increases to the eventual constant $k+1, \quad k_{1} \leqq k+1$. Thus $\left(b^{k+1}: c^{m}\right) \subset\left(b^{k_{1}}: c^{m}\right) \subset$ (b). By Lemma 1 (symmetrically) $\left(b^{n}: c^{m}\right)=\left(b^{k+1}: c^{m}\right)=b^{\prime}\left(b^{k}: c^{m}\right) \subset$ $\left(b^{\prime}\right)$. Therefore $a \in\left(b^{n}: c^{m}\right) \subset\left(b^{\prime}\right)$ and so $b^{k} x=b^{k}\left(a / b^{n}\right) \in R$ since $n=k+j$. Thus $b^{k} R^{(1)} \subset R$.

The last two paragraphs prove the final statement of the 
theorem. However if $C(b, c,-)$ is bounded we now have $b^{k} R^{(1)} \subset R$ with $k$ as above. This shows that $R^{(1)}$ is a finitely generated $R$-module.

Finally suppose that $R^{(1)}$ is a finitely generated $R$-module. Let $J=\left\{r \in R \mid r R^{(1)} \subset R\right\}$. It is easy to see either $\operatorname{Rad} J=M$ or (in case $R^{(1)}=R$ ) $J=R$. Let $k$ be the least integer such that $M^{k} \subset J$. (If $J=R$ we have $k=0$.) We claim that $C(b, c,-)$ is bounded by $k+1$. For any $m \geqq 0$ and $a \in\left(b^{k+1}: c^{m}\right), a / b^{k+1} \in R^{(1)}$ by Lemma 8 . However $b^{k} \in$ $M^{k} \subset J$ so that $b^{k} R^{(1)} \subset R$. In particular $b^{k}\left(a / b^{k+1}\right) \in R$ giving $a \in$ (b). Thus $\left(b^{k+1}: c^{m}\right) \subset(b)$ for all $m \geqq 0$. This shows that $C(b, c,-) \leqq$ $k+1$.

COROllary 10. $R$ is unmixed if and only if $R^{(1)}$ is a finitely generated $R$-module.

Proof. Immediate from Proposition 6 and Theorem 9.

Remark. The result in Corollary 10 is reported in $[2,3.4 .1 \Leftrightarrow$ 3.4.5]. However as is noted in the paragraph preceding [2, 3.4] the result has been known for quite some time. This then allows another proof of Proposition 6, for the known result in Corollary 10 together with Theorem 9 immediately yields Proposition 6.

Our major use of Theorem 9 is the following. For $R$ unmixed we see that $C(b, c,-)$ is eventually $k+1$ where $k$ is the least integer such that $b^{k} R^{(1)} \subset R$. As the definition $R^{(1)}=\cap R_{P}$ over all height 1 primes $P$ does not involve $c$, the condition $b^{k} R^{(1)} \subset R$ involves only $b$ and not c. Thus the constant which $C(b, c,-)$ eventually becomes depends only on $b$. By Lemma 3 and symmetry, the constant which $S(b, c,-)$ eventually becomes depends only on $c$.

Definition. Let $(R, M)$ be a 2-dimensional local unmixed domain. For $0 \neq e \in M$, let ${ }^{*} e$ be the constant which $S(d, e,-)$ eventually becomes, with $d$ any element such that $d, e$ is a system of parameters.

Corollary 11. Let $R$ be unmixed. Then $S(b, c,-)$ eventually equals ${ }^{*} c$ and $C(b, c,-)$ eventually equals ${ }^{*} b+1$.

Proof. Immediate from the definitions, Lemma 3, and symmetry.

REMARK. By Lemma 2, $R$ is Macaulay if and only if $S(b, c,-)$ is identically 0 , or equivalently, $C(c, b,-)$ is identically 1 . By Theorem 9 we see that $R$ is Macaulay if and only if $R^{(1)}=R$. (This is well known and easily proved directly.) In this case Theorem 9 also says that ${ }^{*} b=0$ for all $0 \neq b \in M$. 
EXAMPLE. If $R$ is as in $[2,5.2 .8]$ it is easy to verify that $M R^{(1)} \subset R$ but $R^{(1)} \neq R$. Thus for $0 \neq b \in M, \quad b R^{(1)} \subset R$ but $b^{0} R^{(1)} \not \subset R$. By Theorem 9 and Corollary $11,{ }^{*} b=1$ for all $0 \neq b \in M$.

Knowing that for $R$ unmixed, $S(b, c,-)$ eventually equals ${ }^{*} c$, we ask how long it takes to become that constant. The next result says that $S(b, c, n)={ }^{*} c$ by the time $n={ }^{*} b$. (We do not claim that this is the least $n$ for which $S(b, c, n)={ }^{*} c$.) ${ }^{*} b+1$.

THeorem 12. For $R$ unmixed, $S\left(b, c,{ }^{*} b\right)={ }^{*} c$ and $C\left(b, c,{ }^{*} c\right)=$

Proof. Suppose that $S\left(b, c,{ }^{*} b\right)=k$. To show that $k={ }^{*} c$, the eventual constant value of $S(b, c,-)$, we must show that $S\left(b, c,{ }^{*} b+j\right)=$ $k$ for all $j \geqq 1$. Suppose not. As $S(b, c,-)$ is monotonically increasing, we then have some $j \geqq 1$ with $S\left(b, c,{ }^{*} b+j\right)=l>k$. By Corollary 11, $C(b, c,-)$ eventually becomes ${ }^{*} b+1$. Therefore for some $m \geqq l$, $C(b, c, m)={ }^{*} b+1$ giving $\left(b^{* b+1}: c^{m}\right) \subset(b)$. Since $m \geqq l,\left(b^{* b+1}: c^{l}\right) \subset$ $\left(b^{* b+1}: c^{m}\right) \subset(b)$. By Lemma 1, $\left(b^{* b+1}: c^{l}\right)=b^{\prime}\left(b^{* b}: c^{l}\right)$. However $S\left(b, c,{ }^{*} b\right)=k<l$ implies that $\left(b^{* b}: c^{k}\right)=\left(b^{* b}: c^{l}\right)$. Thus

$$
\left(b^{* b+\jmath}: c^{l}\right)=b^{\prime}\left(b^{* b}: c^{l}\right)=b^{\prime}\left(b^{* b}: c^{k}\right) \subset\left(b^{* b+\jmath}: c^{k}\right) \subset\left(b^{* b+1}: c^{l}\right),
$$

the last two inclusions being trivial. As the extremes of this expression are identical, we conclude that $\left(b^{* b+l}: c^{k}\right)=\left(b^{* b+1}: c^{l}\right)$. This clearly contradicts that $S\left(b, c,{ }^{*} b+j\right)=l>k$ and shows that $S\left(b, c,{ }^{*} b+j\right)=k$ for all $j \geqq 1$. Thus ${ }^{*} c=k=S\left(b, c,{ }^{*} b\right)$. Now by symmetry, $S\left(c, b,{ }^{*} c\right)={ }^{*} b$, so that ${ }^{*} b+1=S\left(c, b,{ }^{*} c\right)+1=C\left(b, c,{ }^{*} c\right)$ by Lemma 3 .

For $R$ unmixed we strengthen Lemma 8 .

\section{Corollary 13. Let $R$ be unmixed. Then}

$$
R^{(1)}=\left\{a / b^{* b} \mid a \in\left(b^{* b}: c^{* c}\right)\right\}
$$

Proof. One inclusion is given by Lemma 8. Therefore take $x \in$ $R^{(1)}$ and write $x=a / b^{n}$ with $a \in\left(b^{n}: c^{m}\right)$ for some $n$ and $m$. We first show that we may take $m={ }^{*} c$. Let $S(b, c, n)=l$. Then clearly $l \leqq{ }^{*} c$, so that $\left(b^{n}: 1\right) \subset\left(b^{n}: c\right) \subset \cdots \subset\left(b^{n}: c^{l}\right)=\left(b^{n}: c^{l+1}\right)=\cdots=\left(b^{n}: c^{*}\right)=$ $\cdots$ Obviously this means $\left(b^{n}: c^{m}\right) \subset\left(b^{n}: c^{* c}\right)$ and so $a \in$ $\left(b^{n}: c^{* c}\right)$. Thus we may take $m={ }^{*} c$.

Now if $n<^{*} b$, say ${ }^{*} b=n+j$ for some $j \geqq 1$. Then $x=a / b^{n}=$ $a b^{\prime} / b^{* b}$ and $a b^{\prime} \in b^{\prime}\left(b^{n}: c^{* c}\right) \subset\left(b^{n+1}: c^{* c}\right)=\left(b^{* b}: c^{* c}\right)$, the inclusion being trivial. In this case we are done. The case $n={ }^{*} b$ is 
immediate. Finally, assume that $n>{ }^{*} b$ and write $n={ }^{*} b+i$ with $i \geqq 1$. By Theorem 12, $C\left(b, c,{ }^{*} c\right)={ }^{*} b+1$, so that $\left(b^{* b+1}: c^{* c}\right) \subset$ (b). By Lemma 1, $\left(b^{* b+c}: c^{* c}\right)=b^{i}\left(b^{* b}: c^{* c}\right)$. Therefore $a \in\left(b^{n}: c^{* c}\right)=\left(b^{* b+1}: c^{* c}\right) \quad$ implies that $a=b^{\prime} a^{\prime}$ with $a^{\prime} \in\left(b^{* b}: c^{* c}\right)$. Since $x=a / b^{n}=b^{i} a^{\prime} / b^{* b+i}=a^{\prime} / b^{* b}$, we are done.

Corollary 14. $R$ is unmixed if and only if $\{S(d, e,-) \mid d$, e is a system of parameters $\}$ is finite.

Proof. Assume that $R$ is unmixed. Then by Corollary $10, R^{(1)}$ is a finitely generated $R$-module. Let $J=\left\{r \in R \mid r R^{(1)} \subset R\right\}$. It is clear from the last sentence of Theorem 9 and Corollary 11 that for $0 \neq e \in M$, ${ }^{*} e$ is the least integer such that $e^{* e} \in J$. As either $\operatorname{Rad} J=M$ or $J=R$, for some $k \geqq 0, M^{k} \subset J$. Thus ${ }^{*} e$ is bounded by $k$. That is $\left\{{ }^{*} e \mid 0 \neq e \in\right.$ $M$ \} is finite. Now $S(d, e, m)$ only takes values between 0 and ${ }^{*} e$, and equals ${ }^{*} e$ for all $m \geqq * d$. Therefore it is clear that for $R$ unmixed, $\{S(d, e,-) \mid d, e$ is a system of parameters $\}$ is finite.

Conversely suppose that $R$ is not unmixed but that $\{S(d, e,-) \mid d, e$ is a system of parameters $\}$ is finite. We will derive a contradiction. As $R$ is not unmixed, each $S(d, e,-)$ is unbounded and monotonically increasing and so has the value 1 at most finitely many times. Thus for some $n \geqq 1$ we have $S(d, e, n)>1$ for all of the finitely many functions $S(d, e,-)$. Let $b, c$ be a system of parameters and let $S(b, c, n)=$ $k$. Then $\left(b^{n}: c^{k}\right)=\left(b^{n}: c^{k+1}\right)=\cdots$. Let $d=b$ and $e=c^{k}$. Clearly $d$, $e$ is a system of parameters and $\left(d^{n}: e\right)=\left(d^{n}: e^{2}\right)=\cdots$. Thus $S(d, e, n) \leqq 1$, contradicting our choice of $n$.

Question. If $R$ is unmixed, Theorem 12 says that $S\left(b, c,{ }^{*} b\right)={ }^{*} c$ and $S\left(c, b,{ }^{*} c\right)={ }^{*} b$. We ask if the converse holds? That is, if $n \geqq 1$, $m \geqq 1$ and $S(b, c, n)=m$ and $S(c, b, m)=n$, is $R$ unmixed (recall $S(b, c, 0)=0$ always so we disallow $n=m=0$ ). Our next result is a little too weak for this, but is strong enough to have interesting corollaries.

THEOREM 15. If for some $n$ and $m, S(b, c, n)=m$ and $S(c, b, m)<$ $n$, then $R$ is unmixed, $S(b, c, n)={ }^{*} c$ and $S(c, b, m)={ }^{*} b$.

Proof. Let $S(c, b, m)=l<n$. Then $\left(c^{m}: b^{l}\right)=\left(c^{m}: b^{l+1}\right)=\cdots=$ $\left(c^{m}: b^{n-1}\right)=\left(c^{m}: b^{n}\right)=\cdots$. By Lemma 1, $\left(b^{n}: c^{m}\right) \subset(b)$. However $S(b, c, n)=m$ gives $\left(b^{n}: c^{m}\right)=\left(b^{n}: c^{m+1}\right)=\cdots$. Thus $\left(b^{n}: c^{m+i}\right)=$ $\left(b^{n}: c^{m}\right) \subset(b)$ for all $i \geqq 0$. Therefore $C(b, c, m+i) \leqq n$ for all $i \geqq$ 0 . By Theorem $6, R$ is unmixed, and by Corollary 11 we also have ${ }^{*} b+1 \leqq n$. By the fact that $S(b, c,-)$ is monotonically increasing and 
by Theorem $12, m=S(b, c, n) \geqq S\left(b, c,{ }^{*} b\right)={ }^{*} c$. On the other hand $m=S(b, c, n)$ clearly implies that $m \leqq{ }^{*} c$. Thus ${ }^{*} c=m=$ $S(b, c, n)$. Finally by Theorem $12,{ }^{*} b=S\left(c, b,{ }^{*} c\right)=S(c, b, m)$.

Corollary 16. Suppose that $R$ is not unmixed. If $S(b, c, n) \leqq m$ then $S(c, b, m) \geqq n$ and $S(c, b, m+1) \geqq n+1$. If also $S(c, b, m)=n$ then $S(b, c, n)=m$.

Proof. Let $S(b, c, n)=m_{1} \leqq m$. We first show that $S(c, b, m) \geqq$ $n$. If not, since $S(c, b,-)$ is monotonically increasing we would have $S\left(c, b, m_{1}\right) \leqq S(c, b, m)<n$. Applying Theorem 15 to $n$ and $m_{1}$ would give that $R$ is unmixed, a contradiction. Thus $S(c, b, m) \geqq n$. We now get $S(c, b, m+1) \geqq n+1$ since if $S(c, b, m+1) \leqq n$, by what we have just done we would have $S(b, c, n) \geqq m+1$ contradicting our assumption. Finally, if $S(c, b, m)=n$ by what we have already done, we have $S(b, c, n) \geqq m$. Combining this with the hypothesis gives $S(b, c, n)=m$.

Remark. Consider the question asked before Theorem 15. If its answer is affirmative then the final sentence in the statement of Corollary 16 is inapplicable. For $n>0$ and $m>0$ it would be impossible to have $S(c, b, m)=n, S(b, c, n)=m$ and $R$ not unmixed. Thus if the question has an affirmative answer, then the first conclusion of Corollary 16 can be strengthened to read ' $S(c, b, m) \geqq n+1$ for $m>0$ '.

Corollary 16 represents all this author knows concerning these functions when $R$ is not unmixed. However there is another class of rings as yet unmentioned in this paper. Recall that $R$ is quasi-unmixed if in the completion of $R$ each minimal prime of zero-divisors has depth equal to $\operatorname{dim} R$. Ferrand and Raymond have constructed a 2dimensional local domain $(R, M)$ which is quasi-unmixed but not unmixed [1, Proposition 3.3]. It is known in our case that $(R, M)$ (2dimensional) is quasi-unmixed if and only if $R^{(1)}$ is integral over $R$. One wonders if quasi-unmixed can be characterized in terms of the functions $S(b, c,-)$ ?

\section{REFERENCES}

1. D. Ferrand and M. Raymond, Fibres Formelles d'un Anneau Local Noetherian, Ann. Sci. École Norm. Sup., 3 (1970), 295-311.

2. L. J. Ratliff, Jr., A theorem on prime divisors of zero and characterizations of unmixed local domains, Pacific J. Math., (to appear).

Received June 1, 1976. 



\section{Pacific Journal of Mathematics}

Vol. 68, No. 1

March, 1977

Richard Julian Bagby, On $L^{p}, L^{q}$ multipliers of Fourier transforms . .......

Robert Beauwens and Jean-Jacques Van Binnebeek, Convergence theorems in

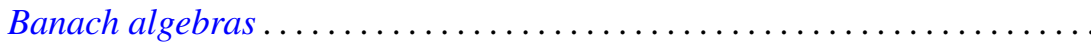

James Cyril Becker, Skew linear vector fields on spheres in the stable

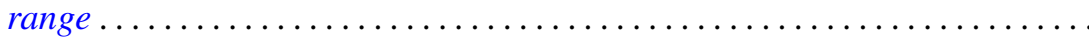

Michael James Beeson, Continuity and comprehension in intuitionistic formal

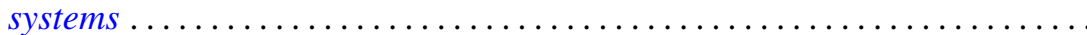

James K. Deveney, Generalized primitive elements for transcendental field

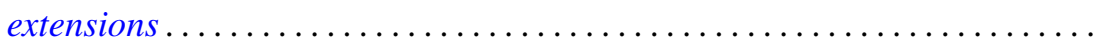

Samuel S. Feder, Samuel Carlos Gitler and K. Y. Lam, Composition properties

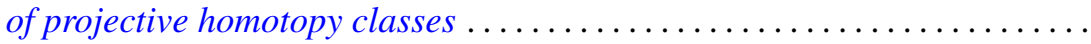

Nathan Jacob Fine, Tensor products of function rings under composition ......

Benno Fuchssteiner, Iterations and fixpoints . . . . . . . . . . . . . .

Wolfgang H. Heil, On punctured balls in manifolds

Shigeru Itoh, A random fixed point theorem for a multivalued contraction

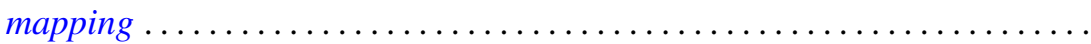

Nicolas P. Jewell, Continuity of module and higher derivations . . . . . . ......

Roger Dale Konyndyk, Residually central wreath products . . . . . . . . . . .

Linda M. Lesniak and John A. Roberts, On Ramsey theory and graphical

parameters.

Vo Thanh Liem, Some cellular subsets of the spheres.

Dieter Lutz, A perturbation theorem for spectral operators

P. H. Maserick, Moments of measures on convex bodies ... . . .

Stephen Joseph McAdam, Unmixed 2-dimensional local domains . .

D. B. McAlister and Norman R. Reilly, E-unitary covers for inverse semigroups...

William H. Meeks, III and Julie Patrusky, Representing codimension-one

homology classes by embedded submanifolds . . .

Premalata Mohapatro, Generalised quasi-Nörlund summability . .

Takahiko Nakazi, Superalgebras of weak-*Dirichlet algebras .

Catherine Louise Olsen, Norms of compact perturbations of operators .

William Henry Ruckle, Absolutely divergent series and isomorphism of

subspaces. II.

Bernard Russo, On the Hausdorff-Young theorem for integral operators .

Arthur Argyle Sagle and J. R. Schumi, Anti-commutative algebras and

homogeneous spaces with multiplications ............

Robert Evert Stong, Stiefel-Whitney classes of manifolds .

D. Suryanarayana, On a theorem of Apostol concerning Möbius functions of

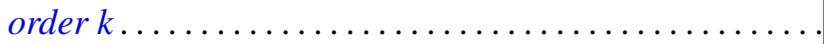

Yoshio Tanaka, On closedness of $C$ - and $C^{*}$-embeddings . . 